\title{
Primera evidencia de circulación de Chlamydophila psittaci en Colombia: posible riesgo de salud pública
}

\section{First evidence of Chlamydophila psittaci circulation in Colombia:} a possible public health risk

Santiago Monsalve, Jorge Miranda y Salim Mattar

Instituto de Investigaciones Biológicas del Trópico. Facultad de Medicina Veterinaria, Universidad de Córdoba. Montería, Colombia. mattarsalim@hotmail.com

Recibido 19 Septiembre 2010/Enviado para Modificación 21 Marzo 2011/Aceptado 3 Abril 2011

\section{RESUMEN}

Objetivo Establecer la seroprevalencia de Chlamydophila psittaci en aves del género Amazona spp y en trabajadores de algunos zoológicos y CAV (centros de atención y valoración de fauna silvestre).

Metodología Se analizaron 138 sueros de aves del género Amazona spp, 24 sueros de otras especies de aves y 39 sueros humanos por ELISA indirecta. Se utilizó el antígeno RMOMP (major outer membrane protein of Chlamydophila psittaci). Para el conjugado de aves se utilizo una anti-lgG de turkey-chicken marcado con biotina para el conjugado humano se utilizo una anti-lgG marcada con peroxidasa. Los sueros fueron diluidos 1:100.

Resultados De los 138 sueros de aves del género Amazona spp 118 (85\%) resultaron positivos. La seroprevalencia por región fue la siguiente: CAV Torre cuatro de Caldas 36 (90\%), Zoológico de Barranquilla 14 (87\%), CAV Montería 28 (85\%), Zoológico de Cali $21(84 \%)$ y CAV Victoria del Oriente Caldense 19 (79\%). En humanos la seroprevalencia total fue del $78 \%(30 / 39)$ la distribución fue la siguiente: CAV Montería 9 trabajadores (100\%), Zoológico de Barranquilla 9 (90\%), CAV Torre 4 de Caldas 4 trabajadores (80\%), CAV Victoria del Oriente Caldense 3 (75\%) y 5 (45\%) trabajadores del Zoológico de Cali. Conclusiones La alta seroprevalencia de Chlamydophila psittaci en aves $(86,4 \%)$ y humanos $(78 \%)$ representa la primera evidencia de la circulación de este microorganismo en Colombia, la presencia del agente etiologico de la ornitosis podría representar un riesgo de salud publica.

Palabras Clave: Chlamydophila psittaci, zoonosis, psitacosis, Colombia (fuente: DeCS, BIREME).

\section{ABSTRACT}

Objective To establish the seroprevalence of Chlamydophila psittaci in birds of Amazona spp genus and workers from some zoos and CAV's (centers for attention and evaluation of wildlife) of Colombia. 
Methods We analyzed 138 sera from birds of the genus Amazona spp, 24 sera from other species of birds and 39 human sera by indirect ELISA. RMOMP was used as antigen (major outer membrane protein of Chlamydophila psittacl). For the conjugate of birds it was used an anti-turkey-chicken IgG labeled with biotin, for the human conjugate we used an anti-lgG labeled with peroxidase. Sera were diluted 1:100.

Results Of the 138 sera from birds of the genus Amazona spp 118 (85\%) were seropositive. Regional seroprevalence was as follow: Caldas CAV Torre cuatro 36 (90\%), CAV's Monteria 28 (85\%), Barranquilla's Zoo 14 (87\%), Cali's Zoo 21 (84\%) and from the CAV Victoria del Oriente Caldense 19 (79\%) sera were seropositive. Regarding seroprevalence in humans, 30 of the $39(78 \%)$ were seropositive, regional seroprevalence was as follow: Montería 9 (100\%) workers, Barranquilla's Zoo 9 (90\%), CAV Caldas Tower four 4 (80 \%), Cali's Zoo 5 (45\%) and CAV Caldense Victoria del oriente 3 (75\%) were seropositive.

Conclusions The high seroprevalence of Chlamydophila psittaci in birds (86,4\%) and humans (78\%) showed the first evidence of circulation of this microorganism in Colombia; the circulation of the etiological agent of psittacosis may represent a public health risk.

Key Words: Chlamydophila psittaci, zoonoses, psittacosis, Colombia (source: $\mathrm{MeSH}$, NLM).

$\mathrm{L}$

as aves silvestres que permanecen cautivas, pueden representar un riesgo para las poblaciones humanas debido a que son reservorios de Chlamydophila psittaci, una bacteria intracelular Gram negativa y que es el agente causal de la ornitosis o psitacosis y representa un riesgo potencial de transmisión zoonótica.

Chlamydophila psittaci es aislada en aves, el contacto entre fauna silvestre y poblaciones humanas por cacería, consumo o como mascotas podría incrementar el riesgo de la transmisión de la zoonosis por Chlamydophila psittaci. Igualmente la transmisión de aves silvestres por la tenencia ilegal de psitacidos en fincas puede conllevar a disminución en la producción y aumento de la mortalidad de aves de corral (2). Las personas que frecuentemente entran en contacto con aves silvestres tienen una mayor predisposición a contraer la infección. Los síntomas humanos son variables e inespecíficos, semejantes a los encontrados en afecciones virales de tipo gripal (fiebres, mialgias, cefaleas, diarrea). No obstante, el cuadro clínico se puede complicar y desencadenar encefalitis, neumonías y endocarditis (Tabla 1). También se han reportado la aparición de infecciones bacterianas secundarias luego de contraer Chlamydophila psittaci (1-6). 
Las aves infectadas liberan la bacteria a través de las heces, orina, exudados respiratorios y oculares, produciéndose la dispersión del mismo a través de las corrientes de aire (Tabla 1). La ingestión de la bacteria conlleva a la infección de las células epiteliales. Pavos, patos, y varios tipos de aves domésticas también pueden ser afectados por diferentes serovariedades de Chlamydophila psittaci (7) (Tabla 1).

Tabla 1. Potencial zoonótico de Chlamydophila psittaci (8)

\begin{tabular}{|c|c|c|c|c|c|c|}
\hline \multicolumn{2}{|c|}{ Hospedero } & \multicolumn{2}{|c|}{ Patología en animales } & \multirow{2}{*}{$\begin{array}{c}\text { Ruta de } \\
\text { transmisión } \\
\text { en humanos }\end{array}$} & \multicolumn{2}{|c|}{ Patología en humanos } \\
\hline Principal & Ocasional & $\begin{array}{l}\text { Signos } \\
\text { clínicos } \\
\end{array}$ & $\begin{array}{c}\text { Enfermedad } \\
\text { severa }\end{array}$ & & $\begin{array}{c}\text { Enfermedad } \\
\text { típica }\end{array}$ & $\begin{array}{c}\text { Enfemedad } \\
\text { severa }\end{array}$ \\
\hline Aves & $\begin{array}{l}\text { Perros, } \\
\text { caballos, } \\
\text { cerdos }\end{array}$ & $\begin{array}{l}\text { Hipertermia, } \\
\text { anorexia, } \\
\text { letargia y } \\
\text { diarrea }\end{array}$ & $\begin{array}{c}\text { Conjuntivitis, } \\
\text { neumonía, } \\
\text { pericarditis y } \\
\text { muerte }\end{array}$ & Inhalación & $\begin{array}{c}\text { Cuadro } \\
\text { sintomático } \\
\text { similar a la } \\
\text { gripa }\end{array}$ & $\begin{array}{c}\text { Endocarditis, } \\
\text { encefalitis, } \\
\text { neumonía y } \\
\text { muerte }\end{array}$ \\
\hline
\end{tabular}

Chlamydophila psittaci es un patógeno que se encuentra actualmente subestimado en nuestro país. En Estados unidos por ejemplo, la bacteria está catalogada como un agente bioterrorista, la cual ha sido clasificada de esta manera por su alta capacidad de transmisión, dispersión y virulencia. Igualmente han sido determinadas altas tasas de morbilidad-mortalidad humana cuando no se aplican los tratamientos antibióticos apropiados, y por la incapacidad de crear inmunidad permanente ante la presencia del patógeno (8).

En Colombia, a pesar de la legislación existente y el control que ejercen las autoridades ambientales, el tráfico de fauna sigue siendo un problema de gran magnitud, sin embargo, por su naturaleza ilegal no se sabe su verdadero alcance, su impacto sobre las poblaciones animales y las consideraciones zoonóticas y de salud pública que se puedan generar de esta situación.

En muchas ocasiones el estado médico, genético o comportamental impide las reintroducciones o liberaciones inmediatas de fauna silvestre, por lo tanto, como única opción para el bienestar animal, es su permanencia temporal en centros de valoración o ser albergados en instituciones zoológicas de forma definitiva (9).

Las aves del orden psitaciforme, principalmente los ejemplares que hacen parte de las especies de loras (Amazona spp), guacamayas (Ara spp), pericos (Brotogeris jugularis), cotorras (Aratinga pertinax) y cascabelitos (Forpus conspicillatus) corresponden al mayor porcentaje de animales que hacen 
parte del proceso del tráfico de fauna silvestre para uso como mascota en hogares de particulares (10). Los ingresos de estos animales por decomisos y entregas voluntarias a la CVS (Corporación Autónoma de los Valles del Sinú y San Jorge) son altos, los ejemplares del orden psitaciforme correspondieron al $57 \%(n=397)$ de los ingresos totales en el año 2009 (11).

En Colombia por su ubicación en el trópico, las zoonosis son frecuentes pero aun falta investigación en el tema. Existe también un subregistro de estas zoonosis y específicamente se desconoce la presencia de la psitacosis. Según informes técnicos del ministerio de salud colombiano una de las enfermedades con riesgo alto y relacionado con la ganadería y otras áreas pecuarias es la ornitosis (12). Existen otros patógenos que causan sintomatología similar a Chlamydophila psittaci; uno de ellos es Coxiella burnetii, causante de la fiebre $\mathrm{Q}$, del cual también se demostró una seroprevalencia del 26,6 \% en los departamentos de Córdoba y Sucre en trabajadores del campo (13).

El objetivo de este estudio fue establecer la seroprevalencia de Chlamydophila psittaci en aves del género Amazona spp y en trabajadores de algunos zoológicos y centros de atención y valoración de fauna silvestre (CAV) de Colombia.

\section{MATERIALES Y MÉTODOS}

Tipo de estudio

Descriptivo prospectivo de corte transversal que se llevo a cabo entre 2008 y 2010.

Estudio de campo, muestreo y tamaño de muestra

Se incluyeron 138 ejemplares de aves del género Amazona spp ( $A$. amazónica, A. ochrocephala, A. autumnalis y A. farinosa) y 39 trabajadores de Zoológicos y CAV de Colombia distribuidos de la siguiente manera: 
Tabla 2. Distribución geográfica y número de muestras obtenidas

\begin{tabular}{llcc}
\hline \multicolumn{1}{c}{ Region geografica } & $\begin{array}{c}\text { Humanos } \mathrm{n}=39 \\
(50)^{*}\end{array}$ & $\begin{array}{c}\text { Amazona sp } \\
\mathrm{n}=138(145)^{\star \star}\end{array}$ \\
\hline 1 & CAV-CVS $^{1}$ Montería & 9 & 33 \\
2 & Zoológico de Cali & 11 & 25 \\
3 & CAV Torre 4 Corpocaldas $_{4}$ & 5 & 40 \\
4 & Zoológico de Barranquilla & 10 & 16 \\
5 & CAV Victoria Corpocaldas-VVS $^{2}$ & 4 & 24 \\
\multicolumn{2}{c}{ Total } & 39 & 138 \\
\hline
\end{tabular}

*Población total de humanos trabajadores en CAV y Zoológicos de Colombia estudiados; ** Población total de aves del género Amazona spp en CAV y Zoológicos de Colombia; ${ }^{1}$ Corporación de los valles del Sinú y San Jorge; ${ }^{2}$ Corporación Valles de Caldas.

En el caso de los ejemplares del género Amazona spp se tomaron 100 individuos, de 145 aves en total de CAV y zoologicos, se utilizó una frecuencia del $16 \%$ basado en los resultados obtenidos en estudios de seroprevalencia en psittacidos en Brasil (14), un error máximo permisible del $1 \%$ y un intervalo de confianza del $99,99 \%$, con estos datos se obtuvo una muestra de 47 ejemplares. Sin embargo, se estudiaron 138 animales en total. Con respecto al tamaño de la poblacion de humanos, se estableció previamente el censo se trabajadores de los CAV y zooógicos y se encontró una población de 50 trabajadores, se tomo una frecuencia del $16 \%$ como valor promedio similar a estudios previos (23-25) un error estadístico del $1 \%$ con un intervalo de confianza del 99,99\%, se obtuvo una muestra de 32 personas sin embargo, se incluyeron 39 individuos.

Toma de muestras

El protocolo realizado fue el de restricción física y química y dependió de las condiciones de cada animal y de cada institución En algunos centros se practicó anestesia con clorhidrato de ketamina intramuscular $(20 \mathrm{mg} / \mathrm{kg}$ en la zona muscular pectoral) con el fin de inmovilizar para posteriormente extraer la muestra necesaria. En otras instituciones solo se utilizó la restricción física para la toma de la misma. Luego de la captura y en algunos casos por medio de anestésicos, se tomó de $0,5 \mathrm{~cm}$ a $1 \mathrm{ml}$ de sangre en la vena alar, basílica o humeral-carpocubital, con jeringa de 2 cc y aguja \#23 o \#25 y se colocó en tubos sin anticoagulante. La sangre se centrifugó durante 5 minutos a 10000 $\mathrm{g}$, el suero se conservó congelado a $-20^{\circ} \mathrm{C}$ hasta su análisis. En las personas se realizó la extracción de la sangre en tubos al vacio sin anticoagulante y se obtuvo el suero y se conservo de igual forma que en los animales. 
ELISA para Chlamydophila psittaci

Se realizó una ELISA indirecta, el antígeno rMOMP (major outer membrane protein de Chlamydophila psittaci) fue fijado en las placas de ELISA diluido 1:40 en coating buffer. Los sueros de animales y humanos se diluyeron 1:100 en PBS con $3 \%$ BSA y $0,05 \%$ de tween 20 y se adiciono $100 \mu \mathrm{l}$ en cada pozo. Las placas fueron incubadas a $37^{\circ} \mathrm{C}$ por 1 hora, después de este periodo fueron lavadas 3 veces con PBS. El conjugado para aves fue una IgG anti-turkey/anti-chicken marcado con biotina y peroxidasa, para conjugado humano se utilizo una IgG anti-Humana marcada con peroxidasa $100 \mu \mathrm{l}$ en cada pozo. La placa se incubo a $37^{\circ} \mathrm{C}$ por 1 hora y lavada 3 veces con PBS. Como sustrato se utilizo $50 \mu \mathrm{ABTS} / \mathrm{H}_{2} \mathrm{O}_{2}$. Los controles positivo y negativo, los conjugados y el antigeno fueron suministrados por la Universidad de Ghent (Belgica), la ELISA utilizada en esta trabajo, no es comercial y se usa con fines investigativos.

Aspectos éticos

El proyecto garantizó que los animales no sufrieran durante la obtención de las muestras, y no se realizó ningún procedimiento que pusiera en riesgo la salud de los animales. La obtención de las muestras se realizó previa anestesia del paciente o por medio de una restricción física adecuada, y se administró bajo estricta vigilancia del veterinario del centro correspondiente. No se muestrearon animales que por su tamaño, senectud o estado de salud pusieran su vida en peligro, la obtención de las muestras se llevó a cabo acorde a las condiciones del sitio y manteniendo las normas de bioseguridad establecidas para tal fin. Se tuvo en cuenta la Resolución No. 008430 de 1993 (4 de octubre de 1 993, artículo 87, literales c, g y h) del Ministerio de Salud de la República de Colombia. Para cumplir con los requisitos de la legislación sobre la investigación científica en diversidad biológica, que involucra alguna o todas las actividades de recolección, captura, caza, pesca, manipulación del recurso biológico y su movilización en el territorio nacional, el Instituto de Investigaciones Biológicas del Trópico solicitó y obtuvo la aprobación de todos los miembros de la ACOPAZOA y de las Corporaciones Regionales Autónomas de donde se tomaron las correspondientes muestras de conformidad con el Decreto 309 artículo $2^{\circ}$ de 2000 del Ministerio del Medio Ambiente en la República de Colombia. Los investigadores de este estudio conocían los "Principios éticos de la experimentación animal" enunciados por el Internacional Council for Laboratory Animal Science (ICLAS) (http:/ /dels.nas.edu/ilar_n/ilarhome/). 


\section{RESULTADOS}

De los 138 sueros de aves del género Amazona spp 118 (85\%) resultaron positivos. La seroprevalencia por región fue la siguiente: Zoológico de Cali 21 (84 \%), CAV Torre cuatro de Caldas 36 (90\%), CAV Victoria del Oriente Caldense 19 (79 \%), CAV Montería 28 (85 \%) y Zoológico de Barranquilla $14(87 \%)$.

En humanos 30 de 39 sueros $(78 \%)$ reaccionaron al antígeno, la distribución fue la siguiente: 5 trabajadores del Zoológico de Cali 5 (45\%), CAV Torre 4 de Caldas 4 (80 \%), CAV Victoria del Oriente Caldense 3 (75 \%), CAV Montería 9 (100 \%) y Zoológico de Barranquilla 9 sueros (90\%) (Figura 1) (Tabla 3).

Figura 1. Áreas geográficas estudiadas para establecer la prevalencia de Chlamydophila psittaci

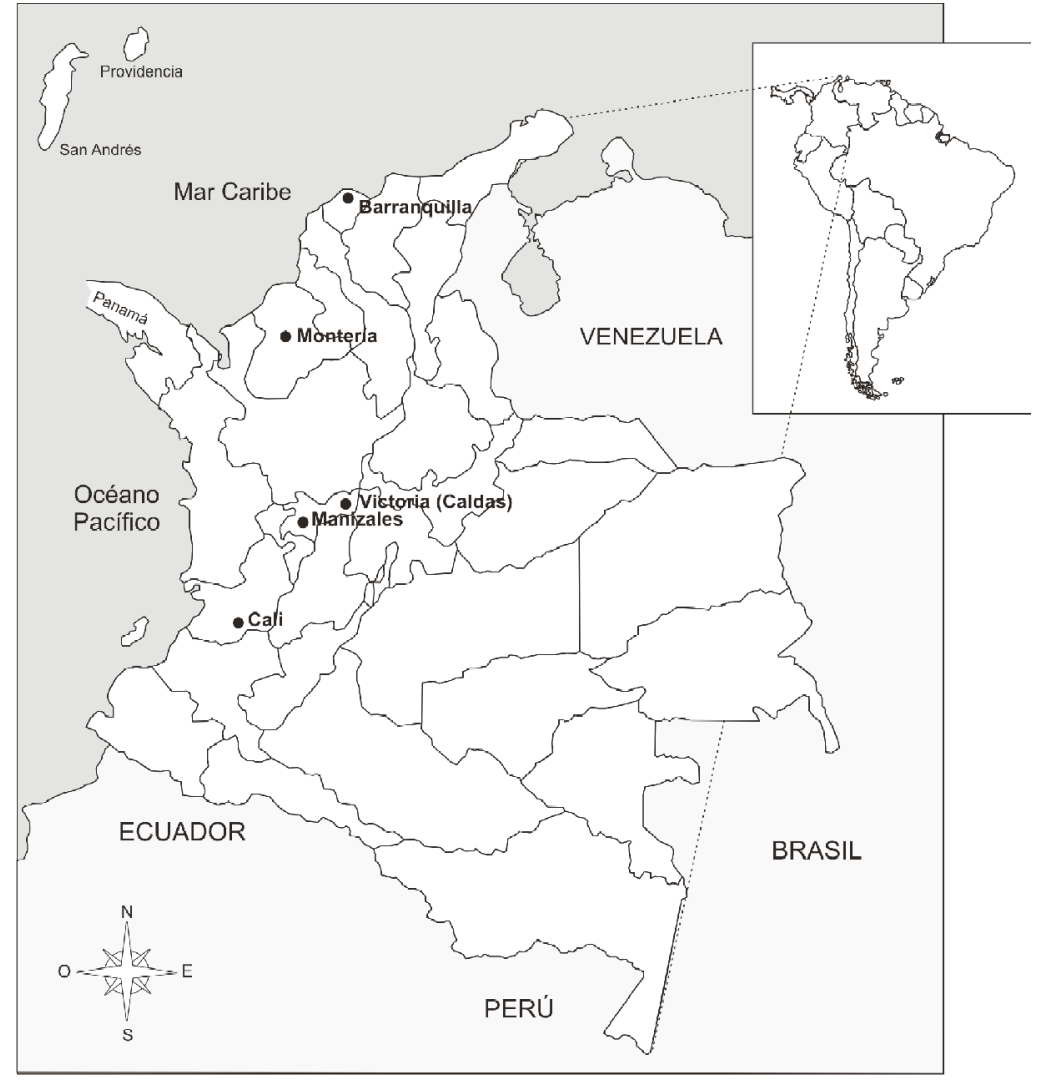


Tabla 3. Prevalencia de Chlamydophila psittaci en aves y humanos en diferentes áreas geográficas de Colombia

\begin{tabular}{|c|c|c|c|c|}
\hline \multirow[b]{2}{*}{ ZOOLÓGICO O CAV (GPS) } & \multicolumn{2}{|c|}{ Loros } & \multicolumn{2}{|c|}{ Humanos } \\
\hline & Número & $\%$ & Número & $\%$ \\
\hline $\begin{array}{l}\text { CAV-T4 Manizales (latitud norte 04눙' } 20^{\prime \prime} \\
\left.\text { longitud oeste } 75^{\circ} 55^{\prime} 45^{\prime \prime}\right)\end{array}$ & 36 & 90 & 4 & 80 \\
\hline $\begin{array}{l}\text { Zoológico Barranquilla (Norte } 11^{\circ} 00^{\prime} 38^{\prime \prime} \\
\left.\text { Oeste } 74^{\circ} 47^{\prime} 52^{\prime \prime}\right)\end{array}$ & 14 & 87 & 9 & 90 \\
\hline $\begin{array}{l}\text { CAV- Montería (Latitud 848'13.976"' } \\
\text { longitud -7550’41.755") }\end{array}$ & 28 & 85 & 9 & 100 \\
\hline Zoológico de Cali (Norte 10200 Este 8200) & 21 & 84 & 5 & 45 \\
\hline $\begin{array}{l}\text { CAV Victoria (Caldas)) (latitud norte } \\
\left.04 \div 48^{\prime} 20^{\prime \prime} \text { - longitud oeste } 75 \circ 55^{\prime} 45^{\prime \prime}\right)\end{array}$ & 19 & 79 & 3 & 75 \\
\hline & Promedic & 85 & Promedio & 78 \\
\hline
\end{tabular}

\section{DISCUSIÓN}

Este es el primer estudio en Colombia sobre la circulación de Chlamydophila psittaci en aves y humanos, la alta seroprevalencia encontrada permite de forma preliminar acercarse al entendimiento del ciclo epidemiológico de la ornitosis.

Algunos estudios en psittacidos muestran prevalencias variables en vida silvestre en diferentes especies (5). Existen diversas opiniones sobre las investigaciones en condiciones in situ de la seroprevalencia del patógeno. Un estudio realizado en la zona del Pantanal en Brasil determinó la seroprevalencia de loras del género Amazona spp en un 6,3\%, y en la especie guacamaya azul (Anodorhynchus hyacinthinus) en un $37 \%$ (14). Por otra parte, en estudios en condiciones ex situ como en Brasil, se estudió la seroprevalencia en ejemplares de loras del género Amazona spp de tres colecciones de zoocriaderos y se determinó una seroprevalencia entre un 16 a un $56 \%$ (15). En contraste, en Bolivia en la zona del Gran Chaco, no se hallaron anticuerpos contra clamidia en loras de frente azul (Amazona aestiva) (16). En Peru, en el Parque Nacional del Manú (17) tampoco se encontró seropositividad para Chlamydophila psittaci en pericos y cotorras (Aratinga weddelli y Brotogeris sanctithomae). Es probable que la ausencia de anticuerpos se deba a las condiciones en vida silvestre de donde se capturaron los ejemplares estudiados y posiblemente a la altitud y variabilidad del clima en esas regiones durante el año. 
La seroprevalencia en aves que permanecen en cautiverio tiende a ser mayor que en ejemplares silvestres. En un estudio realizado en Australia en muestras conjuntivales, cloacales y de orificios nasales tomadas en cacatuas silvestres resultaron negativos por PCR para la detección de Chlamydophila psittaci (18). En otro estudio realizado en Eslovenia con la misma especie en condiciones ex situ se determinó una seroprevalencia del 15,2 \% (19). Las condiciones de estrés por confinamiento que ocasionan una liberación de catecolaminas endógenas de forma crónica, podria sobrellevar a que los ejemplares aviares se inmunodepriman y aumentar asi la susceptibilidad a la infección (20).

En nuestro estudio la seropositividad fue más alta que en otros paises en condiciones in situ en el mismo género animal (Tabla 4).

Tabla 4. Seroprevalencia de Chlamydophila psittaci en ejemplares aviares

\begin{tabular}{|c|c|c|c|}
\hline Especie infectada & Condiciones (pais) & $\begin{array}{l}\text { Seroprevalencia } \\
\text { en loros \% }\end{array}$ & Referencia, año \\
\hline Guacamaya azul & in situ (Brasil) & 37 & $(14), 2006$ \\
\hline Psittacidos & in situ (Brasil) & $6,3-37,8$ & (14), 2006 \\
\hline Psittacidos & ex situ (Brasil) & $16-56$ & (15), 2002 \\
\hline Cacatuas & in situ (Australia) & 0 & (18), 1999 \\
\hline Pericos australianos & in situ (Eslovenia) & 15,2 & (19), 2007 \\
\hline Gaviotas & in situ (Islas Faroe) & 10 & (21), 2006 \\
\hline Amazona spp & ex situ (Colombia) & 85 & presente estudio \\
\hline
\end{tabular}

En el presente estudio se determinó que las aves no presentaron signos clínicos de enfermedad respiratoria compatible con Chlamydophila psittaci, sin embargo, hay una relación entre la alta seropositividad encontrada con respecto a instituciones ubicadas en zonas con mayor altitud sobre el nivel del mar y en los centros muestreados de la región Caribe. En ese sentido, en el CAV torre 4 de Manizales, con una altitud de casi $3000 \mathrm{msnm}$, en las aves estudiadas se encontraron altos niveles de estrés; factores que pudieron incidir en la alta seropositividad a Chlamydophila psittaci. Sin embargo, el presente estudio no pudo controlar la variable geografica sobre el origen de las aves ya que existe desconocimiento sobre la procedencia de los loros previamente al ingreso a los CAVs, por lo tanto fue imposible determinar si el patógeno adquirio la infeccion antes o después de su inclusión a la colección de animales de cada institución. Las diferentes especies del género Amazona spp en condiciones in situ hacen parte de pisos térmicos bajos del trópico, y posiblemente se pueden afectar inmunológicamente por el estrés medioambiental cuando son extraídos de la vida silvestre. 
Para la formulación de planes de rehabilitación y liberación de especies aviares amenazadas, para el mantenimiento definitivo en condiciones ex situ o para el manejo sostenible de los especímenes con fines de zoocría, se deben estandarizar las correctas prácticas de manejo médico, con el fin de prevenir la transmisión a humanos y favorecer la reproducción del animal. Estos son aspectos cruciales en la conservación de especies para que existan datos reales en futuros estudios de investigación, conservación y repoblación. Los centros de conservación ex situ que mantengan ejemplares del género Amazona spp en zonas altas sobre el nivel del mar, deberían replantear el mantenimiento de dichas aves por las condiciones adversas medioambientales que podrían ser determinantes en los indicadores de estrés, lo que en consecuencia conduciría a un posible brote de la enfermedad y en la transmisión zoonótica de la misma.

A pesar de esto la mortalidad causada por Chlamydophila psittaci en vida silvestre ha sido poco reportada. Solo existen pocos estudios donde se identificaron brotes mortales en algunas especies de patos y de gaviotas in situ (21).

De otro lado, la seroprevalencia del $78 \%$ encontrada en humanos es alta, comparada con los resultados en otros estudios; como en Gran Bretaña donde se reportó una seroprevalencia del $3 \%$ en veterinarios y cuidadores de aves (22). Otro trabajo en Brasil determinó una seroprevalencia del 23,9 \% y 4,7 \% por medio de dos pruebas diferentes de diagnostico en trabajadores de zoológicos y veterinarios (23). En Sicilia (Italia) se determinó una seroprevalencia del 14,9\% en trabajadores del campo (24). En Estados Unidos el rango de prevalencia de infecciones zoonóticas en médicos veterinarios estuvo entre el 13,2 \% al 64,5\%, diferencia explicada posiblemente por la variedad de especies implicadas en el ejercicio profesional. De los veterinarios de zoológicos en EEUU el 30,2 \% fueron hospitalizados por diferentes tipos de enfermedades zoonóticas, algunas por psitacosis (25) (Tabla 5).

En el presente estudio en humanos existio una correlación entre el mayor porcentaje de seropositividad y la zona geográfica donde están ubicados los CAVs y zoologicos. En ese sentido en la poblacion humana de la región Caribe se determino una mayor seropositividad por Chlamydophila psittaci que en el resto trabajadores del país. Tambien regionalmente la seroprevalencia de las aves del Caribe es la segunda en el estudio por lo que coinciderian con una posible circulacion del microoragnismo en poblaciones expuestas. El zoológico de Cali ha sido catalogado como uno de los mejores 
centros de conservación ex situ, y la seropositividad baja encontrada difiere con los resultados encontrados en otros centros de conservación. Las condiciones de mantenimiento en cautiverio, los protocolos de manejo en el bienestar y los programas de prevención de riesgos profesionales quizá sean las razones por las cuales exista una menor seropositividad en humanos y aves en dicha institución que en otros centros de conservación.

Tabla 5. Seroprevalencia de Chlamydophila psittaci en humanos

\begin{tabular}{lccc}
\hline \multicolumn{1}{c}{ Grupo humano afectado } & País & $\begin{array}{c}\text { Seroprevalencia } \\
\text { en humanos \% }\end{array}$ & Referencia, año \\
\hline Veterinarios & Inglaterra & 3 & $(22), 1999$ \\
Trabajadores de Zoológicos & Brasil & 23,9 & $(23), 2009$ \\
Veterinarios & Brasil & 4,7 & $(23), 2009$ \\
Trabajadores del campo & Italia & 14,9 & $(24), 2007$ \\
Veterinarios de Zoológicos & EEUU & $13,2-64,5$ & $(25), 1998$ \\
Trabajadores y veterinarios de Zoológicos & Colombia & 78 & Presente estudio \\
\hline
\end{tabular}

Por otra parte, en el presente estudio no se puede descartar reactividad cruzada con Chlamydophila pneumoniae o Chlamydia trachomatis en humanos, ya que los epitopes MOMP son compartidos con otras especies de Chlamydiaceae. No obstante, de esta reacción cruzada, los resultados del estudio son contundentes con respecto a la presencia de Chlamydophila psittaci ya que las aves no se infectan con especies humanas como $C$. pneumoniae y C. trachomatis.

Por otra parte, el tráfico de fauna silvestre de estas especies puede desencadenar en serios problemas de salud pública. Los ejemplares del género amazona no deberían ser considerados como mascotas hasta no realizar más estudios con respecto a la posible transmisión de enfermedades que estas aves puedan transmitir. Igualmente se hace necesario profundizar en la formulación de protocolos de manejo en cautiverio de la especie, tanto médico como nutricional y biológico. En ese sentido con base a los resultados de este trabajo, es posible en Colombia comenzar una labor de educación a las personas tenedoras de mascotas y a la comunidad médica del país. EL aporte de este trabajo no es solo fundamental para el ministerio de la proteccion social y al INS, tambien lo es para los medicos tratantes de enfermedades infecciosas por que deben considerar en el espectro diagnostico a Chlamydophila psittaci.

En conclusión, se determinó que existe la circulación de Chlamydophila psittaci en aves del género Amazona que permanecen en confinamiento y en una poblacion de humanos expuesta que trabajan con aves, lo que podría 
generar un problema de salud pública. La psitacosis humana podría ser una importante causa de riesgo ocupacional en aquellas personas que se encuentren en contacto con aves. Los trabajadores deberían ser informados de los riesgos de la enfermedad, su mecanismo de transmisión y del uso de equipos de protección adecuados (26)

Agradecimientos: A ACOPAZOA, a la Fundación Botánica y Zoológico de Barranquilla, a la Fundación Zoológica de Cali, a las Corporaciones Regionales Autónomas (Corpocaldas y CVS), y a la VVS por ser colaboradoras del proyecto por la autorización en la toma de muestras. A Daisy Varompay Ph.D de la Universidad de GENT (Bélgica) por los antígenos y reactivos de ELISA. A la Universidad de Córdoba (CIUC) por la financiación del proyecto código: FMV-0608 y al Convenio Universidad de Córdoba-Gobernación de Córdoba CODECYT. Los resultados de este trabajo hacen parte de la tesis de maestría en Ciencias Ambientales de S. Monsalve.

\section{REFERENCIAS}

1. Roberts JP, Grimes JE. Chlamydia shedding by four species of wild bids. Avian Dis. 1978; 22 : 698-706.

2. Vanrompay D, Ducatelle R, Haesebrouck F. Chlamydia psittaci infections: a review with emphasis of avian chlamydiosis. Veterinary Microbiology. 1995; 45: 93-119.

3. Wobeser G, Brand CJ. Chlamydiosis in biologists investigating disease occurences in wild waterfowl. Wildl. Sot. Bull. 1982; 10:170-172.

4. Franson JC. Chlamydiosis in gulls from North Dakota: a reminder to consider diseases transmissable to humans when investigating wildlife mortality. Washington DC: US Dept. of Interior, Fish and Wildlife Service Research Information Bulletin. 1987; 47:1-2.

5. Brand CJ. Chlamydial infections in free-living birds. J. Am. Vet. Med. Assoc. 1989; 195:15311535.

6. Gerbermann H, Korbel R. Zum Vorkommen von Chlamydiapsittaci Infektionen bei Greifvogeln ausfreier Wildbahn. Tierarztl. Prax. 1993; 21:217-224.

7. Vanrompay D, Andersen AA, Ducatelle R, Haesebmuck F. Serotyping of European isolates of Chlamydia psittaci from poultry and other birds. J Clin Microbiol. 1993; 31:134-137.

8. Rodolakis A, Mohamad KY. Zoonotic potential of Chlamydophila. Veterinary Microbiology. 2009; 140 (2010) 382-391.

9. Fundación Botánica y Zoológica de Barranquilla. Censo poblacional del tráfico de fauna en el área metropolitana de la ciudad de Barranquilla; 2005.

10. Rodríguez JV, Rojas F, Arzuza DE, Gonzales A. Loros, pericos y guacamayas neotropicales. Conservación Internacional. Serie libretas de campo. 2005; 35-37.

11. Corporación Regional Autónoma de los Valles del Sinú y San Jorge CVS. Informe técnico anual de actividades. CAV convenio 03 CVS Unicórdoba; 2009. p. 9-12.

12. Castaño P. Estudio y análisis del riesgo biológico ocupacional en Colombia. Informe técnico, Ministerio de Trabajo y seguridad social; 1997. p. 9-80.

13. Máttar S, Parra M. Detection of antibodies to Anaplasma, Bartonella and Coxiella in rural inhabitants of the Caribbean area of Colombia. Rev. MVZ Córdoba. 2006; 11(2):781789.

14. Raso TF, Fernandes GH, Robaldo NM, Pinto AA, Chlamydophila psittaci in free-living Bluefronted Amazon parrots (Amazona aestiva) and Hyacinth macaws (Anodorhynchus hyacinthinus) in the Pantanal of Mato Grosso do Sul, Brazil. Veterinary Microbiology. 2006; 117: 235-241. 
15. Raso T de F, Júnior AB, Pinto AA. Discusión: Evidence of Chlamydophila psittaci infection in captive Amazon parrots in Brazil. J Zoo Wildl Med. 2002; 33(2):118-21.

16. Deem SL, Noss AJ, Cuellar RL, Karesh WB. Health evaluation of free-ranging and captive Blue-fronted Amazon parrots (Amazona aestiva) in the Gran Chaco, Bolivia. J. Zoo Wildlife Med 2005; 36: 598-605.

17. Gilardi KV, Lowenstine LJ, Gilard JD, Munn CA. A survey for selected viral, chlamydial, and parasitic diseases in wild dusky-headed parakeets (Aratinga weddellii) and tui parakeets (Brotogeris sanctithomae) in Peru. J. Wildlife Dis 1995; 31: 523-528.

18. McElnea CL, Cross GM. Methods of detection of Chlamydia psittaci in domesticated and wild birds. Aust. Vet. J. 1999; 77: 516-521.

19. Dovc A, Slavec B, Lindtner-Fnific R, Zoroman-Rojs O, Racnik J, Golja J, Vlahovic K. Study of Chlamydophila psittaci outbreak in budgerigars. Bull Vet Inst Pulawy. 2007; 51: 343-346.

20. Greco G, Corrente M, Martella V. Detection of Chlamydophila psittaci in Asymptomatic Animals. J Clin Microbiol. 2005; 43 (10): 5410-5411.

21. Herrmann B, Persson H, Jensen JK, Joensen HD, Klint M, Olsen B. Chlamydophila psittaci in Fulmars, the Faroe Islands. Emerging Infectious Diseases. 2006; 12 (2) 330-332.

22. Gosbell IB, Ross AD, Turner IB. Chlamydia psittaci infection and reinfection in a veterinarian. Aust. Vet. 1999; 77: 511-513.

23. Raso TF, Carrasco AO, Silva JC, Marvulo MF, Pinto AA. Seroprevalence of Antibodies to Chlamydophila psittaci in Zoo Workers in Brazil. Zoonoses and Public Health. 2009; 57: 411-416.

24. Fenga C, Cacciola A, Di Nola C, Calimeri S, Lo Giudice D, Pugliese M, Niutta PP, Martino LB. Serologic investigation of the prevalence of Chlamydophila psittaci in occupationally- exposed subjects in eastern Sicily. Ann Agric Environ Med. 2007; 14: 93-96.

25. Hill D, Langley R, Morrow M. Occupational Injuries and illnesses reported by zoo veterinarians in the United States. Journal of Zoo and Wildlife Medicine. 1998; 29(4):371-385.

26. NASPHV (National Association of State Public Health Veterinarians), Veterinary Infection Control Committee, 2008: Compendium of Veterinary Standard Precautions for Zoonotic Disease Prevention in Veterinary Personnel [Internet]. Disponible en: http:/ /www.nasphv.org/Documents/VeterinaryPrecautions Consultado en: abril 22, 2011. 